\title{
Enhancing IEEE 802.11 MAC in congested environments ${ }^{1}$
}

\author{
Imad Aad ${ }^{\mathrm{a}, *, 2}$, Qiang $\mathrm{Ni}^{\mathrm{b}, 2}$, Chadi Barakat ${ }^{\mathrm{c}}$, Thierry Turletti ${ }^{\mathrm{d}}$ \\ ${ }^{\text {a } E P F L, ~ S w i t z e r l a n d ~}$ \\ ${ }^{\mathrm{b}}$ Hamilton Institute, National University of Ireland Maynooth, Ireland \\ c Planète Group - INRIA Sophia Antipolis, France \\ dPlanète Group - INRIA Sophia Antipolis, France
}

\begin{abstract}
IEEE 802.11 is currently the most deployed wireless local area networking standard. It uses carrier sense multiple access with collision avoidance (CSMA/CA) to resolve contention between nodes. Contention windows $(\mathrm{CW})$ change dynamically to adapt to the contention level: Upon each collision, a node doubles its $\mathrm{CW}$ to reduce further collision risks. Upon a successful transmission, the $\mathrm{CW}$ is reset, assuming that the contention level has dropped. However, the contention level is more likely to change slowly, and resetting the CW causes new collisions and retransmissions before the CW reaches the optimal value again. This wastes bandwidth and increases delays. In this paper we analyze simple slow CW decrease functions and compare their performances to the legacy standard. We use simulations and mathematical modeling to show their considerable improvements at all contention levels and transient phases, especially in highly congested environments.
\end{abstract}

Key words: Wireless communications, IEEE 802.11, MAC, CSMA/CA, Simulations, Markov chains.

\section{Introduction}

* Corresponding author. Present address:

EPFL - I\&C - LCA

BC 246 (Bâtiment BC)

Station 14

CH - 1015 Lausanne

Switzerland

1 Part of this work has been presented at the IEEE PIMRC 2003 conference [14].

2 Part of this work was done while they were with INRIA, France.
Wireless access networks are experiencing a huge success, similar to that of the deployment of the Internet a decade ago. Wireless devices are used almost everywhere to provide cheap, mobile and easy to deploy networks, with or without access to wired infrastructures such as the Internet. Wireless access networks can be grouped into two categories: Centralized or distributed (ad-hoc). Central- 
ized architectures are mainly controlled by a coordinator that grants access to the wireless nodes in its area in a contention-free manner. Alternatively, distributed architectures have no central coordinators: All nodes contend to access the channel using a distributed function. IEEE 802.11 [1-3] is currently the most deployed wireless local area access network (LAN) standard. It supports two access functions, one is centralized at the access point (AP), the other is distributed. The distributed coordination function (DCF) is based on carrier sense multiple access (CSMA)[4] with collision avoidance (CA). Using CSMA/CA, each node differs its transmission to a random time in the future and senses the channel before trying to transmit. Upon each collision, notified by the absence of acknowledgment (ACK) from the destination, the node increases the bound of the random deferring time, called contention window $(\mathrm{CW})$. Increasing the $\mathrm{CW}$ reduces the risk of further collisions, assuming the number of contending nodes is high. Nodes may optionally use request to send / clear to send (RTS/CTS) frames to reserve the channel before the actual data-ACK frame transmissions. Upon each successful transmission, a node resets its CW to $C W_{\min }$ and contends again with low CW values.

Our work in this paper aims to enhance this last point: Upon a successful transmission, a wireless node resets its $\mathrm{CW}$, therefore it takes the risk of experiencing the same collisions and retransmissions until it reaches high $\mathrm{CW}$ values again, wasting time and bandwidth. Assuming that the number of contending terminals changes slowly, this risk is likely to be high. We propose slow CW decrease (SD) functions and evaluate their performance by comparing them to the IEEE 802.11 standard. Simulations and mathematical models show that these functions outperform the legacy 802.11 standard in terms of throughput, delays, jitter and power consumption.
The next section presents the motivations and related work. Section 3 introduces the approach of slow CW decrease (SD) and evaluates its performances from the throughput, delay and jitter point of view, using simulations and mathematical modeling. Section 4 introduces another performance metric, the settling time of SD. Section 5 explores the fairness properties of the proposed SD scheme, then, in Section 6 we analyze its energy savings. We analyze in Section 7 the channel noise effect on the mechanism. In Section 8 we evaluate the implementation costs and we conclude the paper in Section 9.

\section{Motivations and related work}

In a distributed wireless congested environment, a station has no knowledge of the number of contending terminals. The 802.11 standard MAC protocol adapts its CW to the current congested level by doubling its CW upon each collision and by resetting it upon a successful transmission. Doubling the CW assumes a higher congestion level and therefore the need to increase the CW. When a node increases its $\mathrm{CW}$, it reduces the chances of simultaneous transmissions with other nodes, at the cost of more backoff overhead. This reduces collisions and the corresponding retransmission times, therefore improving the throughput. When a node succeeds in transmitting a frame, it assumes that the congestion level has dropped, and therefore it resets its $\mathrm{CW}$ to $C W_{\text {min }}$. However, when a node succeeds in transmitting a frame at a given $C W_{i}$, this does not correspond to a congestion level decrease, but rather to a convenient CW value. Therefore the CW value must be kept large as long as the congestion level remains the same. By resetting the CW, a node takes the risk of experiencing the same collisions and retransmissions until it reaches convenient (high) CW 
values again, wasting time and bandwidth.

To adapt the CW to possible drops of congestion level, we should consider decreasing the CW upon successful transmissions. However, since the congestion level is not likely to drop suddenly, we should consider slow CW decrease (SD) functions. Intuitively, the advantage of SD functions is more collision avoidance during congestion periods, leading to less collisions and retransmissions, which increases throughput and decreases delays. The drawback is keeping high CW values after the congestion level drops, increasing the overhead and decreasing the throughput. This inconvenience is negligible compared to the advantage of SD, since it is very unlikely that the congestion would drop quickly to low levels. In the following sections we propose SD functions and evaluate their performances by comparing them to the actual standard, in different scenarios.

The slow CW decrease was first introduced in MACAW [5], which is an extension to the CSMA [4] and MACA [6] schemes. The main idea of MACAW is to increase the $\mathrm{CW}$ at each collision by multiplying it by 1.5 , and to linearly decrease it by 1 at each successful frame transmission. The approach is called MILD (multiplicative increase, linear decrease). The analysis was restricted to the effect of linear decrease functions on the channel efficiency only. Furthermore, only throughput measures in infrastructure mode were considered. Adhoc topologies and other performance metrics like fairness, delays, resilience to noise, power consumption and settling times were unexplored.

In [7], the slow CW decrease was considered, but from the fairness enhancement point of view. [7] tries to establish local utility functions in order to achieve system-wide fairness, with no explicit global coordination. Then, it "translates" a given fairness model into corresponding backoff-based collision resolution algorithms that probabilistically achieve the fairness objective. These algorithms include different backoff increase/decrease factors. [7] tries to enhance the fairness properties that MACAW [5] and CB-Fair first proposed in [8]. Always aiming to establish fair contention algorithms, [8] uses smooth CW increase and decrease functions. Each station $i$ contends to access the channel in order to send a frame to station $j$ with a probability $p_{i j}$, computed in two ways using time-based and connection-based methods. These methods are pre-established using information broadcast by each station such as the number of logical connections and the contention time.

In this paper we aim to investigate different CW decrease functions from the data rate, delay, response time, fairness and power saving efficiency point of view. Our main contributions are: (i) we propose an efficient multiplicative CW decrease approach to improve the throughput of 802.11 MAC protocol; (ii) we use mathematical modeling and simulation results to evaluate the performance of the SD scheme in different scenarios.

\section{Throughput, delay and jitter analysis}

Consider 100 wireless stations, uniformly distributed in a $100 \mathrm{~m} \times 100 \mathrm{~m}$ square area, that communicate with each other two by two (50 flows). All nodes are within the range of each other, hence no routing protocol is needed. We start the simulation using the network simulator, $n s-2[9]$, at time $t=40$ seconds (s). We increase the number of active flows by one every two seconds. Each transmitting station sends 1050-byte CBR packets every $5 \mathrm{~ms}$ (providing full data rate of $1.6 \mathrm{Mbps})$. At $t=150 \mathrm{~s}$, all traffic sources stop except one. This corre- 


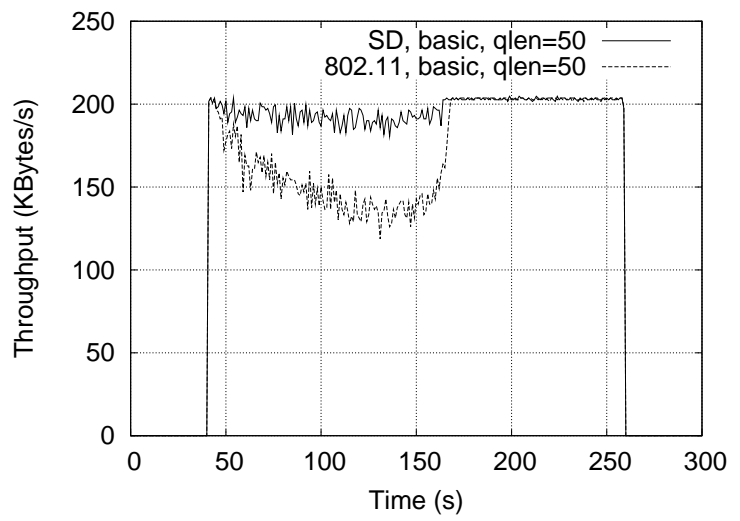

Fig. 1. Total throughput comparison, without RTS/CTS.

sponds to the worst case scenario for SD: large CWs (due to the previously increasing congestion) whereas the channel is not congested at all. At $t=260 \mathrm{~s}$, all sources stop sending data. Optimally, when the number of contending flows $n$ increases, each flow would get $1 / n$ of the available data rate. However, due to the increasing collisions, the actual throughput observed by each flow is lower than this value.

The dashed curve in Figure 1 shows how the total throughput, averaged over one-second intervals, decreases as the number of contending flows increases (e.g. during $t=40 \mathrm{~s} \rightarrow 150 \mathrm{~s}$ ). In fact, after each collision, the source has to wait for a timeout to realize that the frame collided, increases its CW (to reduce further collision risks) then retransmits the frame. After a successful transmission the source resets its CW.

As a node resets its CW after a successful transmission, it "forgets" about the collision experience it had. If all stations keep transmitting with the same data rate, most probably the new transmission will observe the same contention and collisions as before. This can be avoided by keeping some history on the observed collisions: Instead of resetting the CW to $C W_{\text {min }}$, we set the $\mathrm{CW}$ to 0.9 times its previous value (lower bounded by $C W_{\text {min }}$, i.e. $\left.C W_{\text {new }}=\max \left\{C W_{\text {min }}, 0.9 \times C W_{\text {prev }}\right\}\right)$. The solid curve in Figure 1 shows the considerable throughput enhancement we get (up to $37 \%$ ), especially with a high number of active flows (at $t=150 \mathrm{~s}$ ). When we decrease the CW slowly, we waste more time on backoff, but this enhances collision avoidance. Slowly decreasing the CW makes it oscillate around the (theoretic) optimal CW value [10], with no need to estimate the number of contending flows [11]. Furthermore, throughput is more stable, due to lower/smoother variations of CW values. SD is a tradeoff between large CW values and risking a collision followed by the whole frame retransmission. Since the time induced by frame retransmissions is much larger than the backoff time, SD is much better on average. The average overhead due to backoff and retransmissions can be written as:

$E[$ overhead $]=O_{b k o f}(j) \times\left(1-P_{c o l}\right)+O_{\text {retx }+b k o f} \times P_{c o l}$

where $P_{c o l}$ is the probability of a collision, $O_{b k o f}(j)$ is the overhead due to the backoff time at stage $j$ of the successful transmission, and

$$
O_{\text {retx }+b k o f}=\sum_{i=1}^{r}\left(O_{b k o f}(i)+T_{d a t a}\right)
$$

is the overhead due to retransmissions and their corresponding backoffs, $r$ is the number of retransmissions until a successful frame reception, and $T_{\text {data }}$ is the data transmission time in the basic mode.

The worst case for SD would be when we consider high $\mathrm{CW}$ values, but no congestion takes place. This is the case at $t=150 \mathrm{~s}$, when we stop all but one transmission in order to observe the remaining throughput. Figure 1 shows that SD still behaves better than resetting the CW. After a few successful transmissions, SD would reach the $C W_{\min }$ value that 


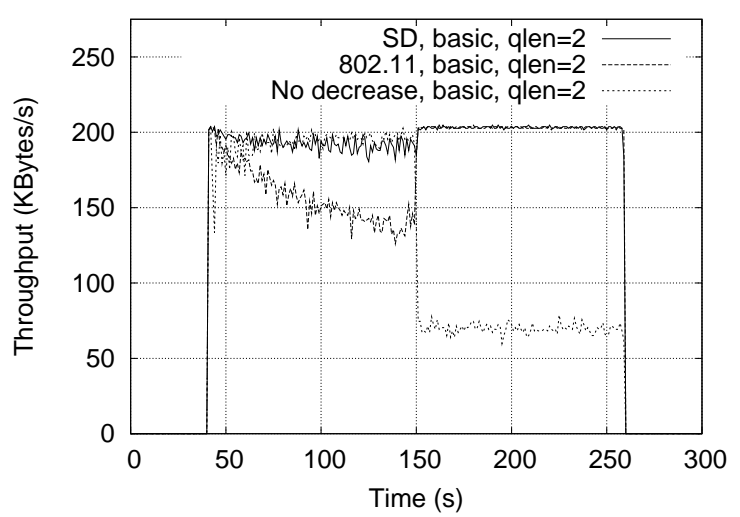

Fig. 2. Total throughput comparison, without RTS/CTS, qlen=2.

the CW reset scheme would have reached directly. Note that the CW reset scheme takes a long time to increase its throughput. In fact, all traffic sources (but one) stop at $t=150 \mathrm{~s}$, but the effect is "shifted" to around $t=168 \mathrm{~s}$. This is due to the residual frames queued in the interfaces of all 49 transmitters (the interface queue length is 50 frames). After stopping the sources, these remaining frames will continue contending to access the channel, which possibly cause collisions.

Consider now the same scenario as before, but with shorter interface queue lengths $(=2)$, in order to remove the effect of smoothly stopping sources and to observe the real overhead due to SD. Figure 2 shows that the above queueing effects are eliminated, and the overhead due to SD can be observed in its worst case (no congestion, high $\mathrm{CW}$ values, i.e. at $t=150 \mathrm{~s}$ ).

This shows that SD performs as well as CW reset scheme at low congestion, even right after high congestion. This can be considered as the response of the SD function to the changing congestion frequency at its maximum, i.e. when the number of contending nodes vary up and down very fast. SD performs as well at lower congestion variations, when the number of transmitting sources moves up and down more slowly.

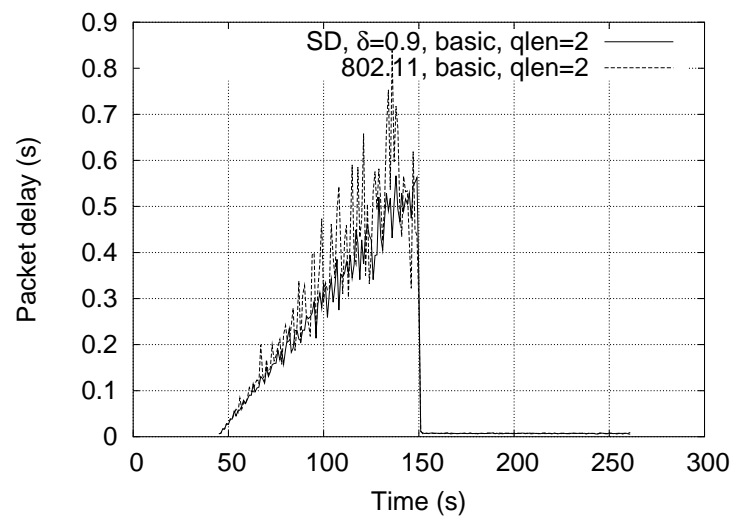

Fig. 3. Packet delays comparison, without RTS/CTS, qlen $=2$.

For comparison, we add a third curve to Figure 2 , showing the overall throughput when we do not decrease the $\mathrm{CW}$ at all, i.e. keeping it at its maximum reached values. The performance decreases considerably at low congestion levels and high CW values, as we can see for the remaining active flow after $t=150 \mathrm{~s}$. This shows that the backoff time cannot be absolutely considered as negligible and must be reduced upon successful transmissions.

Figure 3 shows the delay observed for the same simulation scenarios. We can see how the delay increases with the number of contending nodes for both the SD (solid curve) and the CW reset scheme (dashed curve). SD shows lower delays and jitters. Since the CW decreases slowly, more collisions and retransmissions are avoided, leading to lower average delays. And since the CW varies slowly, keeping better adapted to the actual congestion level, the jitter is lower than the one with the CW reset scheme by tens of milliseconds. The probabilities of a successful transmission change with the CW variation, therefore using sudden CW reset after each successful transmission leads to very high jitters. SD has lower jitters, showing the convenience of this approach typically at high congestion levels. We should note that when we consider longer interface queues (e.g. 50 ), delays become orders of a magnitude 


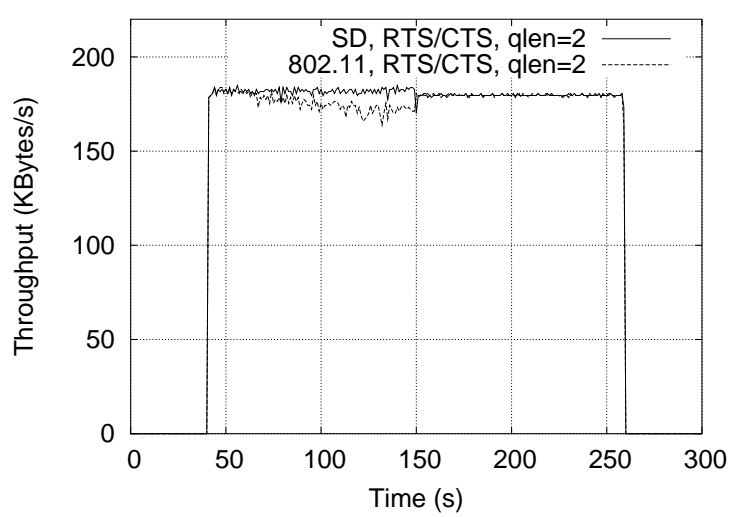

Fig. 4. Total throughput comparison with RTS/CTS.

higher than the delays in Figure 3.

When we use short data frames, the relative gain decreases and SD becomes less efficient: The backoff overhead introduced by SD becomes comparable to the frame payload. To that end, consider the RTS/CTS exchange before a data frame transmission. SD avoids (short) RTS collisions that are less severe. Therefore we observe a low gain of SD over the CW reset scheme.

This can be seen in Figure 4. We observe no gain when the congestion level is low. SD performs as well as the CW reset scheme. At high congestion levels $(t=150 \mathrm{~s})$, we observe a better throughput enhancement. Obviously, RTS/CTS adds overhead and performs less than the basic scheme, whether using SD or CW reset schemes.

In order to evaluate the performance of the SD approach, we introduce two metrics:

- Throughput gain $(G)$ : This is the ratio of the throughput obtained by applying SD over the throughput obtained by applying the CW reset scheme.

- Settling time $\left(T_{l}\right)$ : After a sudden decrease of the number of active stations (e.g. at $t=$ $150 \mathrm{~s}), T_{l}$ is the time it takes a single flow to reach its steady state throughput, with

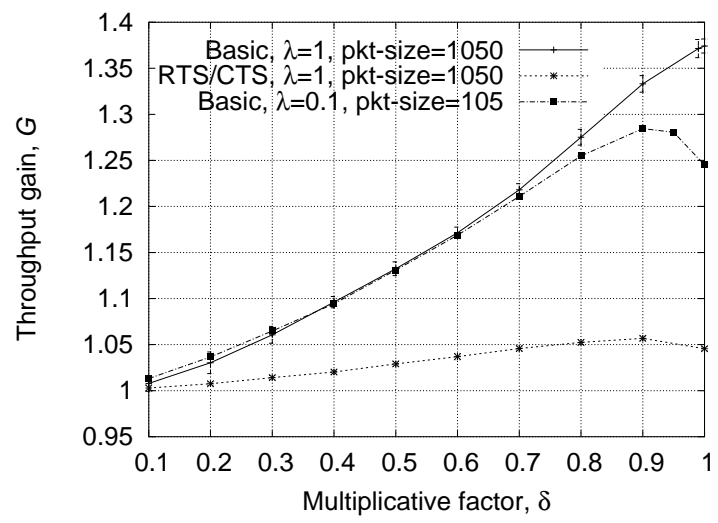

Fig. 5. Throughput gain, $G$, vs. CW decrease factor $\delta$.

small CW values. $T_{l}$ characterizes the system response time that uses slow CW decrease.

In the following we will use different $\mathrm{CW}$ decrease factors $\delta$ and different data rates $\lambda(\lambda$ $=($ source data rate $) /$ (maximum channel capacity) ) to evaluate $G$ and $T_{l}$. Figure 5 shows the throughput gain $G$ function of the CW decrease factor $\delta$. Each point is averaged over 9 simulation runs, and the confidence interval is 95\%. We can see that:

- When $\delta$ decreases, the slow CW decrease becomes closer (resembles more) to the CW reset scheme and shows no enhancement over this last, $(G \rightarrow 1)$.

- However, when the multiplicative factor $\delta$ is high, CW decreases slowly upon each successful frame transmission, still avoiding future collisions and retransmissions, therefore the throughput is higher than with the $\mathrm{CW}$ reset scheme $(G>1)$.

- When using small frame sizes, the throughput gain $(G)$ decreases since collisions have less effect on the total throughput, and the maximum gain $G_{\max }$ is around $\delta_{\max }=0.9$. Beyond this value, the backoff overhead of SD becomes considerable and $G$ starts decreasing.

- As $\lambda$ decreases, the throughput gain $G$ decreases for all values of $\delta$. In fact, when the data rates decrease, we observe fewer colli- 
sions leading to fewer CW increases and CW decreases. Therefore the gain of SD over the CW reset scheme lowers and converges to unity.

- When $\delta=1$, we observe a non-negligible gain $G>1$ when the channel is highly congested (as seen in Figure 5). However, when the channel suddenly becomes less congested, the CW value keeps constantly high, increasing overhead, and decreasing throughput efficiency. For low data rates, this overhead (when $\delta=1$ ) is negligible relative to the idle channel periods between consecutive packets. Therefore the gain $G=1$. However, when $\lambda=1$, this overhead becomes considerable leaving large idle gaps between packets, reducing efficiency, therefore the gain drops to $G=0.48$.

- When using $\delta<1$, the CW size (and thus its overhead) will progressively decrease upon each successful transmission. Therefore the overhead cited above (with $\delta=1$ ) still exists but for a transient period only, the duration of which is a function of $\delta$, the frame data rate $\lambda$ and the corresponding successful transmissions. This transient period is characterized by $T_{l}$, the settling time we defined above, and will be analyzed in section 4 .

\subsection{Mathematical model}

Our analysis is divided into two parts. First we study the behavior of a single mobile station with a SD Markov model, and we compute the stationary probability $\tau$ that the station transmits a packet in a randomly chosen time slot. This probability does not depend on the access mechanisms (with or without RTS/CTS scheme). Second, by studying the events that occur within a time slot, we express the channel throughput as a function of $\tau$ with and without RTS/CTS schemes. We obtain then a system of two equations that we solve for the

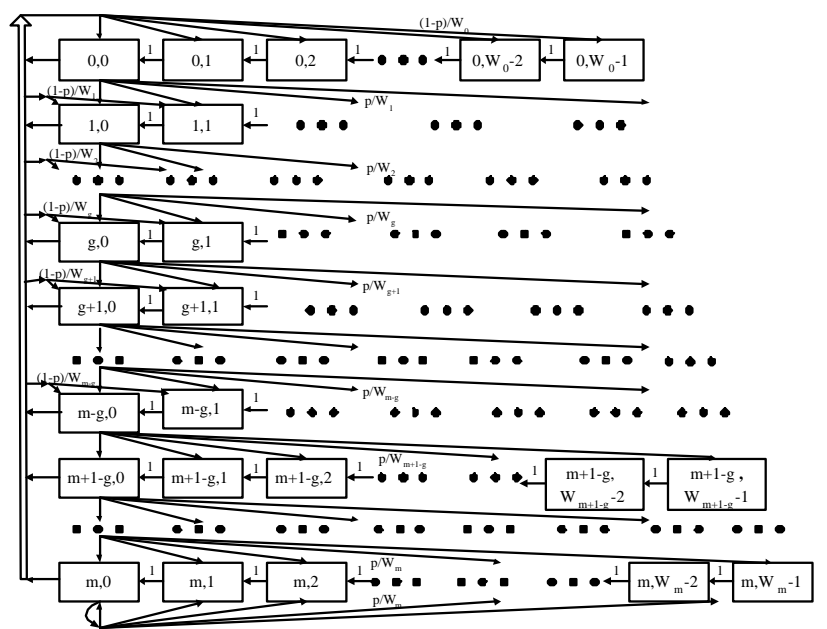

Fig. 6. Markov chain model for the SD scheme. channel throughput by eliminating $\tau$.

\subsubsection{Analysis of the packet transmission probability}

We make the same assumptions as in [10]. A fixed number $n$ of stations is considered and the transmission queue of each station is always nonempty. Each packet has to wait for a random backoff time decrement to zero before transmitting. $p$ denotes the probability that a packet collides and $\sigma$ denotes the time slot duration. A time slot is equal to the IEEE 802.11 time slot $\sigma_{0}$ if no packets are transmitted. If a packet is being transmitted, $\sigma$ is equal to the busy period until the channel is idle again for a time period equal to DIFS. We define two stochastic processes to model the protocol behavior (Figure 6). First, $b(t)$ represents the backoff counter of the time a station has to wait before it can transmit. This process has the range from 0 to the current $\mathrm{CW}$ size. Another stochastic process $s(t)$ is defined as the backoff stage at different CW levels. $s(t)$ scales from 0 to $m$, with $m$ being the maximum CW stage.

With these assumptions, the bi-dimensional stochastic process $\{s(t), b(t)\}$ fulfills the properties of an homogeneous discrete Markov 
chain. The Markovian property does not hold for the process $b(t)$ alone, which depends on the backoff stage history. For simplicity, we write $W_{i}$ instead of $C W_{i}$ and $W_{0}$ instead of $C W_{\min }$. Since the contention window doubles after each collision, we can write $W_{i}=2^{i} \times W_{0}$, where $0 \leq i \leq m$. The maximum backoff stage $m$ is the value such that $C W_{\max }=2^{m} \times W_{0}$. We suppose that the constant decrease factor $\delta$ has a power of two form, $\delta=1 /\left(2^{g}\right)$, where the constant factor $g$ is an integer with $g>0$. This choice of $\delta$ limits the number of states of the Markov chain and simplifies the analysis, without affecting the results. Thus, the new $\mathrm{CW}$ value when a packet is correctly transmitted will be:

$$
W_{i+1}=\max \left(W_{0}, \delta \times W_{i}\right)=\max \left(W_{0}, W_{i-g}\right) .
$$

Consider the transitions of the SD scheme between time slots. For instance, we ignore time slots where the station is transmitting. Figure 6 explains the behavior of the Markov chain. The only non-null one-step transition probabilities are:

$P\{i, k \mid i, k+1\}=1$, for $k \in\left[0, W_{i}-2\right], i \in[0, m]$.

$$
\begin{aligned}
P\{0, k \mid i, 0\}=(1-p) / W_{0}, & \pi_{0,0} & =(1-p) \sum_{j=0}^{g} \pi_{j, 0} . & \\
& \text { for } k \in\left[0, W_{0}-1\right], i \in[0, g-1] . & \pi_{i, 0} & =p \pi_{i-1,0}+(1-p) \pi_{i+g, 0}, 0<i \leq m-g . \\
P\{i-g, k \mid i, 0\}=(1-p) / W_{i-g}, & \pi_{i, 0} & =p \pi_{i-1,0}, & m-g<i<m . \\
& \text { for } k \in\left[0, W_{i-g}-1\right], i \in[g, m] . & p \pi_{m-1,0} & =(1-p) \pi_{m, 0} . \\
P\{i, k \mid i-1,0\}=p / W_{i}, & \Rightarrow \pi_{m, 0} & =\frac{p}{1-p} \pi_{m-1,0}, & i=m .
\end{aligned}
$$$$
\text { for } k \in\left[0, W_{i}-1\right], i \in[1, m] \text {. }
$$$$
P\{m, k \mid m, 0\}=p / W_{m}, \quad \text { for } k \in\left[0, W_{m}-1\right] \text {. }
$$

The first equation in (1) accounts for the fact that the backoff timer has not reached 0 and that it is decremented by 1 at the beginning of each time slot. The second and third equations are specific to the SD scheme. The second equation accounts for the fact that when $\delta \times W_{i}$ is smaller than $W_{0}$, we reset $W_{i}$ to $W_{0}$, and a new backoff is uniformly chosen in the range $\left(0, W_{0}-1\right)$. The third equation accounts for the fact that when $\delta \times W_{i}$ is larger than $W_{0}$, we decrease $W_{i}$ slowly to the new value $W_{i-g}$ and we choose the new backoff counter randomly in the range $\left(0, W_{i-g}\right)$. The fourth and the fifth equations correspond to the cases where a collision occurs.

Let $\pi_{i, k}=\lim _{t \rightarrow \infty} P\{s(t)=i, b(t)=k\}, i \in$ $[0, m], k \in\left[0, W_{i}-1\right]$, be the stationary distribution of the Markov chain. As the Markov chain is ergodic, this distribution exists and is unique. First, we will express all $\pi_{i, k}$ as function of $\pi_{0,0}$, then we will use the normalization equation to solve for $\pi_{0,0}$, and hence for all $\pi_{i, k}$.

From the Markov chain above, we can see that the incoming traffic to stage $i$ from either $(i+$ $g, 0)$ after a successful transmission, or from $(i-1,0)$ after a collision, is uniformly distributed over all possible backoff values at this stage. Afterwards, the counter is decremented by one and finally reaches $(i, 0)$. So, the stationary probability $\pi_{i, 0}$ is given by:

The first equation in (2) accounts for the fact that stage 0 can only be reached from stages $j \leq g$ in the SD scheme, the stages $j>g$ can not directly decrease to stage 0 . The second equation in (2) says that for stages $0<i \leq$ $m-g$, there are two different inputs: From the 
previous stage with collision probability $p$ and from the stage $i+g$ after a successful transmission with a probability $1-p$. For stages $i>m-g$, there will be no input from stages $i+g$, because $i+g$ is bigger than the maximum stage number $m$. For stage $m$, we fall into a special case, since after a collision the contention window remains at this stage.

Now, according to the Markov chain regularities, for each $k \in\left[1, W_{i}-1\right], \pi_{i, k}$ can be written as:

$$
\pi_{i, k}=\frac{W_{i}-k}{W_{i}}\left\{\begin{array}{c}
(1-p) \sum_{j=0}^{g} \pi_{j, 0}, \text { for } i=0 . \\
p \pi_{i-1,0}+(1-p) \pi_{i+g, 0}, \\
\text { for } 0<i \leq m-g . \\
p \pi_{i-1,0}, \text { for } m-g<i \leq m . \\
p\left(\pi_{m-1,0}+\pi_{m, 0}\right), \text { for } i=m .
\end{array}\right.
$$

The ratio before the parentheses accounts for the distribution of probabilities for each state in a stage. When we move in a stage to the right, the probability decreases by $1 / W_{i}$, since we do not get the input of the previous state in the same stage. Thus, we can obtain the relationship between $\pi_{i, k}$ and $\pi_{i, 0}: \pi_{i, k}=\left(W_{i}-\right.$ $k) / W_{i} \times \pi_{i, 0}$. By using (2), we get the term on the right-hand side of the parentheses in (3). Equation (3) then allows us to compute all stationary probabilities as a function of $\pi_{0,0}$ and $p$. Obtaining closed-form expressions does not seem possible, so we proceed by solving the system numerically with Matlab: First we solve formulas in (2) to obtain $\pi_{i, 0}$ that are only dependent on $\pi_{0,0}$ and $p$. Then we plug them into (3) to obtain $\pi_{i, k}$ that are only dependent on $\pi_{0,0}$ and $p . \pi_{0,0}$ is finally computed by using the normalization condition:

$$
1=\sum_{i=0}^{m} \sum_{k=0}^{W_{i}-1} \pi_{i, k}
$$

Now we compute $\tau$, the probability that a station transmits in a time slot. This probability is simply the sum of probabilities of all $(i, 0)$ states,

$$
\tau=\sum_{i=0}^{m} \pi_{i, 0}=f\left(p, W_{0}, g, m\right)
$$

This expression of $\tau$ is a function of $p$, which is unknown. The other three variables $\left(W_{0}, g, m\right)$ have known values. Let us assume independence of all the stations that share the medium, i.e. the probability that a station encounters a contention is independent of the status of the other stations. The $n$ stations are identical so they all transmit packets in a slot time with the same probability $\tau$. Consider that a station transmits a packet in a time slot. $p$ is then the probability that at least one other station transmits a packet in the same slot:

$$
p=1-(1-\tau)^{(n-1)} \text {. }
$$

We obtain a non-linear system of two equations (5) and (6), that can be solved for $p$ and $\tau$. This system certainly has a solution, since (i) the expression of $p$ in (6) is continuously increasing with $\tau$, with $p=0$ for $\tau=0$ and $p=1$ for $\tau=1$, and (ii) the expression of $\tau$ in (5) is continuous with $p$. A sufficient condition for this solution to be unique is that the expression of $\tau$ in (5) is continuously decreasing with $p$, i.e. more contention leads to less transmissions. Our numerical results show that this is always the case and hence a unique solution for our model always exists. 


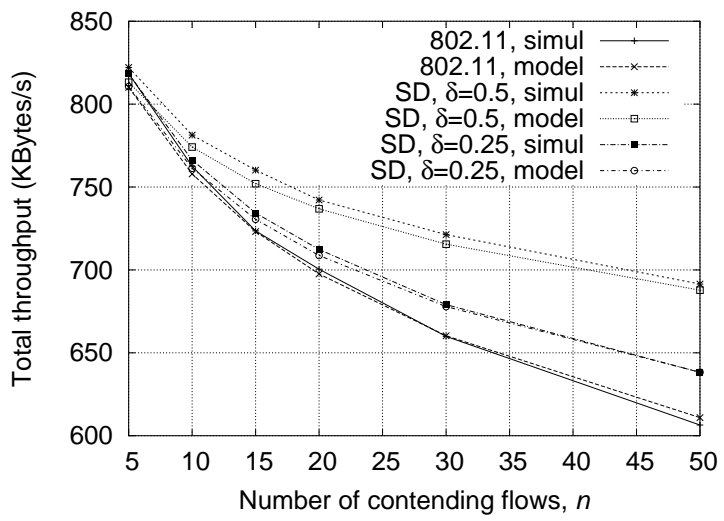

Fig. 7. Throughput of SD and IEEE 802.11 vs. the number of contending flows, model and simulation results.

\subsubsection{Throughput}

Denote by $S$ the throughput per station, that is by definition the average volume of data correctly transmitted by a station in a slot time divided by the average slot time duration. Consider a random time slot, let $P_{t r}$ be the probability that there is at least one transmission in this time slot, and let $P_{s}$ be the probability of one successful transmission given that there is at least one transmission. Note that

$$
P_{t r}=1-(1-\tau)^{n}, \text { and } P_{s}=\frac{n \tau(1-\tau)^{n-1}}{1-(1-\tau)^{n}} .
$$

Hence, $S=\frac{P_{t r} P_{s} E[P]}{\left(1-P_{t r}\right) \sigma+P_{t r} P_{s} T_{s}+P_{t r}\left(1-P_{s}\right) T_{c}}$

where $T_{s}$ is the average time the channel is sensed busy because of a successful transmission, and $T_{c}$ is the average time the channel is sensed busy because of a collision. We use in our analysis the values of $T_{s}$ and $T_{c}$ computed in [10]. Note that the throughput expression (7) does not specify the access mechanism employed. To account for whether RTS/CTS is used, we only need to specify the corresponding values $T_{s}$ and $T_{c}[10]$.

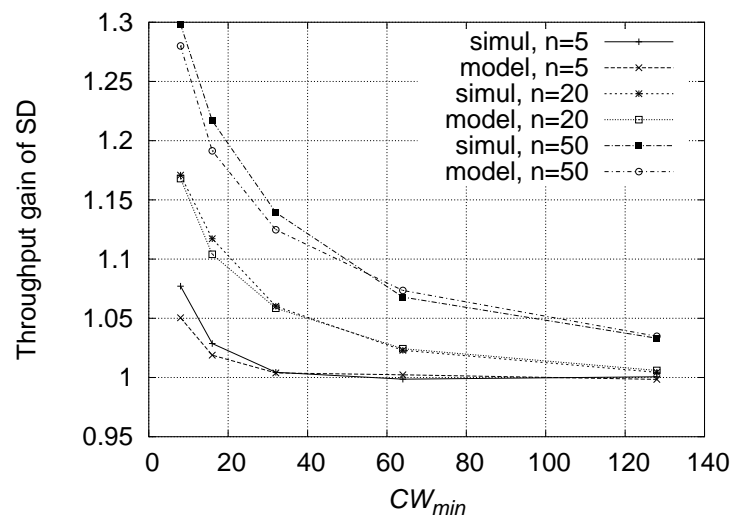

Fig. 8. Throughput gain of SD over IEEE 802.11, vs. $C W_{\min }$, varying the number of contending flows.

Figure 7 shows the throughput model and simulation results for various decrease factors $(\delta)$ and for legacy IEEE 802.11, when we increase the number of contending nodes (basic scheme, 1050-bytes packets, $1 \mathrm{Mbits} / \mathrm{s}$ channel). The model results are quite similar to simulation results. We can see the considerable throughput enhancement we get with high values of $\delta$ and high number of contending nodes. SD throughput gain decreases when the number of contending nodes decreases and when $\delta$ decreases, but it keeps outperforming 802.11.

Figure 8 shows the throughput gain of SD over 802.11 when varying the $C W_{\min }$ and the number of nodes. The simulations and the model show close results: The gain decreases when $C W_{\text {min }}$ increases, since increasing $C W_{\text {min }}$ contributes to collision avoidance, hence the effect of SD decreases. Furthermore, as cited before, this gain increases with the number of contending flows. 


\section{Settling time}

To measure the settling time $T_{l}$, we proceed using another simple scenario ${ }^{3}$ : A single flow is considered. We force the CW to its maximum, 1023, as it would be in highly congested environments. This reduces its throughput considerably. Then we let the CW use SD and the $\mathrm{CW}$ reset schemes respectively, and measure the settling times $T_{l}$.

Figure 9 shows that, as expected, when $\delta$ increases, we need more successful transmissions before the throughput reaches its steady state, that is $T_{l}$ increases. This increase is much higher than linear, especially for high $\delta$ values. The reader should distinguish the settling time $T_{l}$, which concerns throughput stability, from frame transmission delays. In the previous examples, a $T_{l}$ of $100 \mathrm{~ms}$ simply means that 40 consecutive frames must be sent successfully before the throughput reaches its high steady state. However, evaluating the user perception of $T_{l}$ is out of scope of this work.

Choosing the right multiplicative decrease factor $\delta$ is a compromise between having a high throughput gain $G$ and a short settling time $T_{l}$, for the case of sudden congestion decrease. Intermediate $\delta$ values like $0.6-0.9$ would satisfy such a tradeoff. For a smoother congestion decrease, ${ }^{4}$ one would choose higher $\delta$ values to get higher throughput gains, without much care about $T_{l}$.

We also investigated linear SD which showed it can reach the same gain values as multiplicative CW decrease. However, the settling time $T_{l}$ is higher than with multiplicative CW decrease, especially for small linear decrease con-

3 This scenario corresponds to the system response to an impulse input, from the feedback control point of view.

4 In practice, this is hard to predict.

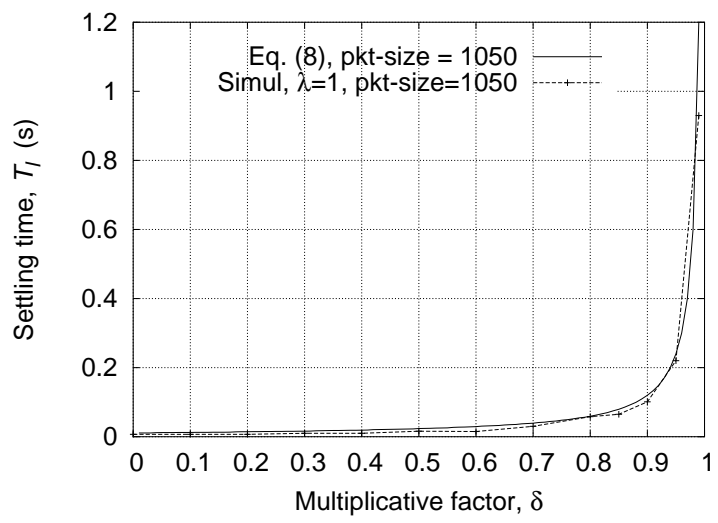

Fig. 9. Settling time $T_{l}$ vs. $\delta$.

stants $(\alpha)$ that would lead to good throughput enhancements.

Finally we should note that in [5], Bharghavan et al. use linear $\mathrm{SD}$ with $\alpha=1$. This surely enhances throughput, like very high $\delta$ values do with multiplicative SD. However, very high $\delta$ values and very low $\alpha$ values would lead to unacceptable settling times $T_{l}$, if one considers a sudden congestion level drop. From the user point of view, high settling time values $\left(T_{l}\right)$ mean longer delays before the user gets the maximum throughput after moving from a highly congested area to a lowly congested area, or when all of his neighbors suddenly stop their transmissions.

It is easy to obtain a closed-form expression for the maximum settling time $T_{l}$. We need to send $l$ consecutive frames successfully, to reach the "optimal" throughput (with $C W_{\text {min }}$ ), i.e.

$$
C W_{\max } \times \delta^{l}=C W_{\min },
$$

therefore $l=\left\lfloor\frac{\ln \left(C W_{\min } / C W_{\max }\right)}{\ln (\delta)}\right\rfloor$, those $l$ frames take an average time

$T_{l}=\sum_{i=0}^{l}\left(T_{s}+O_{b k o f}(i)\right)=(l+1) T_{s}+\frac{C W_{\max }}{2} \sigma \frac{1-\delta^{l+1}}{1-\delta}$,

where $i$ is the transmission attempt number, 
$T_{s}$ is the frame transmission time with its corresponding DIFS, SIFS and ACK transmission time, and $\sigma$ is the time slot duration.

\section{Fairness analysis}

This section is divided into two parts. The first analyzes short-term and long-term fairness of $\mathrm{SD}$. The second analyzes long-term fairness between IEEE 802.11 nodes and SD nodes operating together.

\subsection{Fairness amongst similar nodes}

Before discussing the fairness of the SD scheme, let us check some issues related to fairness in the current IEEE 802.11. To measure fairness, we use Jain's index of fairness $\left(F_{J}\right)[12]$. We consider a given number of accesses (a window) to the channel and compute $F_{J}$ as:

$$
F_{J}=\frac{\left(\sum_{i=1}^{n} \gamma_{i}\right)^{2}}{n \sum_{i=1}^{n} \gamma_{i}^{2}}
$$

where $n$ is the number of nodes and $\gamma_{i}$ is the proportion of successful accesses of node $i$ during the considered window. $F_{J}$ is equal to unity when all nodes equally share the medium, and it is equal to $1 / n$ when a single node monopolizes the channel (in which case $F_{J} \rightarrow 0$ when $n \rightarrow \infty$ ). We compute the average $F_{J}$ by sliding the window through all the simulation time. Figure 10 shows, as in [13], the weak fairness of IEEE 802.11 on the short-term scale. This fairness obviously improves when the window size used for measurement increases.

When we increase the $C W_{\min }$ value, we see that fairness also improves (Figure 10): After a successful transmission, a node (with a

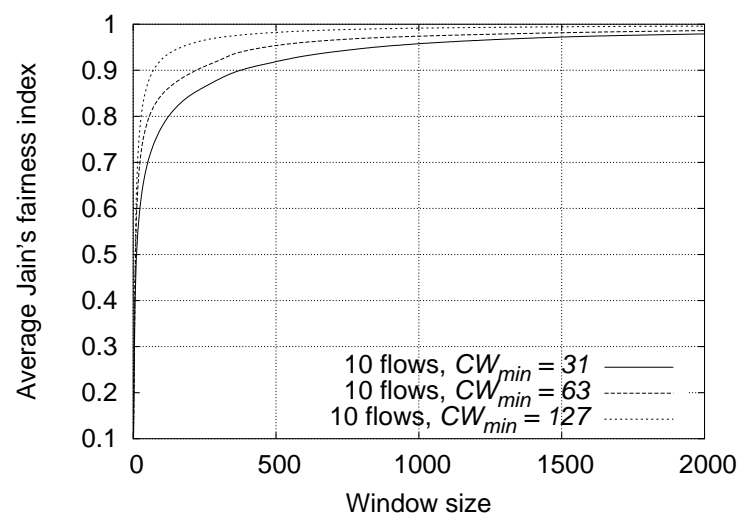

Fig. 10. Fairness of IEEE 802.11 when varying $C W_{\min }$

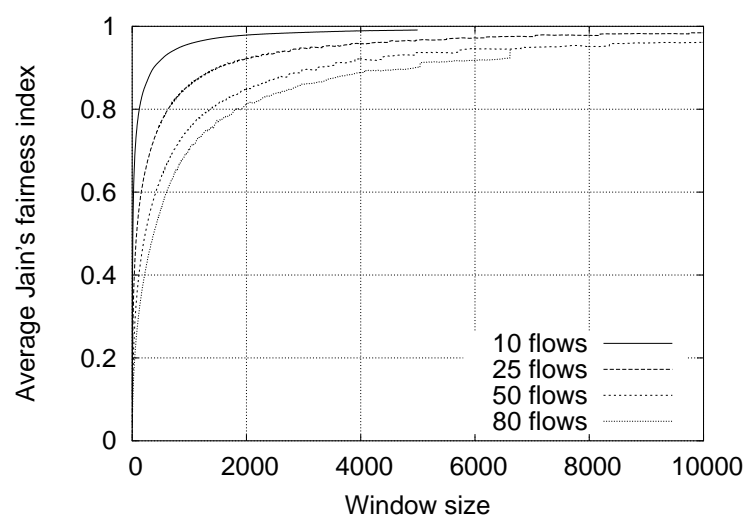

Fig. 11. Fairness of IEEE 802.11 when varying the number of contending nodes

high $C W_{\text {min }}$ ) has a lower probability to access the channel right afterwards, which gives other contending nodes higher probabilities to access the channel, and hence improves the fairness. However, this is not the case when we increase the number of contending nodes (Figure 11):

Indeed, when we increase the number of contending nodes, we increase the collision rate. This increases the risk of having a group of nodes with high CWs (after collisions), whereas other nodes get the chance to transmit several frames more frequently, therefore degrading fairness.

The above aspects of improving fairness with $C W_{\min }$ and degrading it with the number of contending nodes, are combined when we in- 


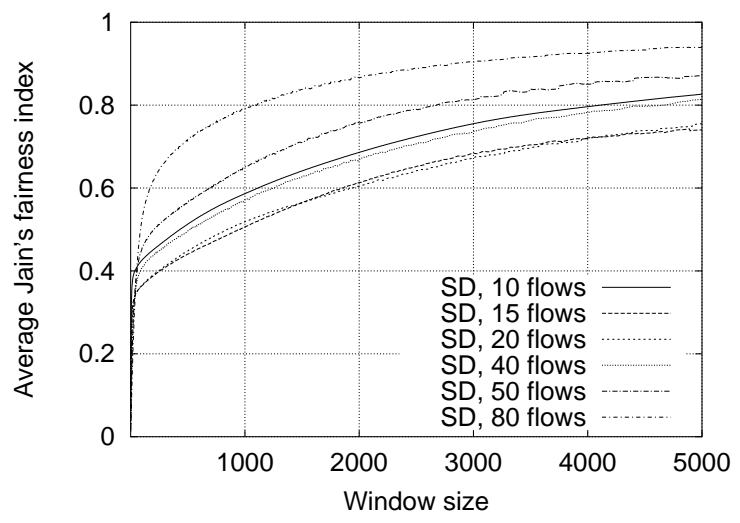

Fig. 12. Fairness of SD when varying the number of contending nodes

crease the number of nodes with SD (Figure 12).

Using SD increases the average CW sizes, which is supposed to improve fairness. However the increasing number of nodes tends to degrade fairness. Therefore, with a fixed multiplicative decrease factor (0.9), we notice that when we increase the number of nodes, fairness decreases down to a given level, then starts increasing. That is the point where large CW sizes compensate the unfairness of the high number of contending flows.

Figure 13 compares the fairness of IEEE 802.11 and SD. For a small number of contending flows, 802.11 is more fair than SD. When we increase the number of flows, the fairness curves of the two schemes become close to each other. For a high number of contending flows, SD shows better fairness than IEEE 802.11.

\subsection{Fairness with legacy IEEE 802.11 nodes}

The main drawback of using SD is the unequal share of data rate it gets when it coexists with IEEE 802.11. Consider the scenario where part of the competing nodes uses IEEE 802.11 and the other part uses SD.

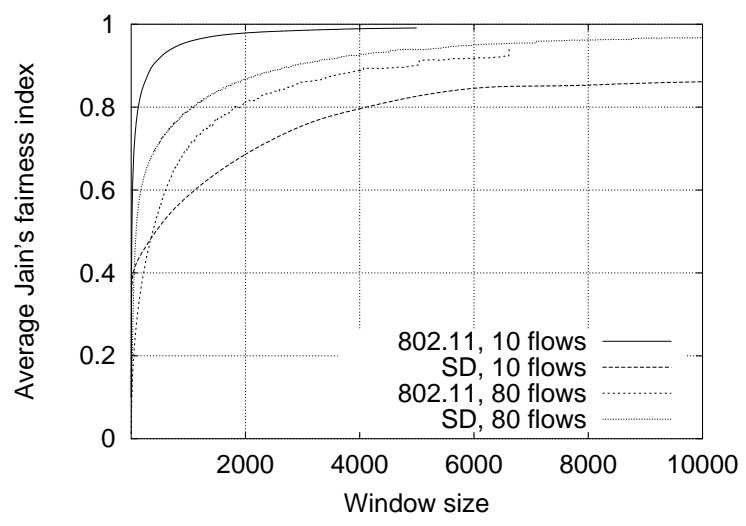

Fig. 13. Comparing fairness of IEEE 802.11 and $\mathrm{SD}$

Let $n_{1}$ be the number of IEEE 802.11 stations, $\tau_{1}$ be the probability that an IEEE 802.11 station transmits in a time slot, $p_{1}$ be the collision probability seen by an IEEE 802.11 station, and let $P_{t r 1}$ be the probability that one IEEE 802.11 transmits packets in the considered time slot (it is the same as $\tau_{1}$ ). $P_{s 1}$ be the probability that one IEEE 802.11 station transmission occurring on the channel is successful. Let $n_{2}, \tau_{2}, p_{2}, P_{t r 2}$ and $P_{s 2}$ be the corresponding values for $\mathrm{SD}$ stations. We keep the same meanings and notations of $T_{s}$ and $T_{c}$ as in 3.1.2. The throughput of one IEEE 802.11 station will be:

$$
S_{1}=\frac{P_{t r 1} P_{s 1} E[P]}{\left(1-P_{t r}\right) \sigma+P_{t r} P_{s} T_{s}+P_{t r}\left(1-P_{s}\right) T_{c}} .
$$

The throughput of an SD station will be:

$$
S_{2}=\frac{P_{t r 2} P_{s 2} E[P]}{\left(1-P_{t r}\right) \sigma+P_{t r} P_{s} T_{s}+P_{t r}\left(1-P_{s}\right) T_{c}},
$$




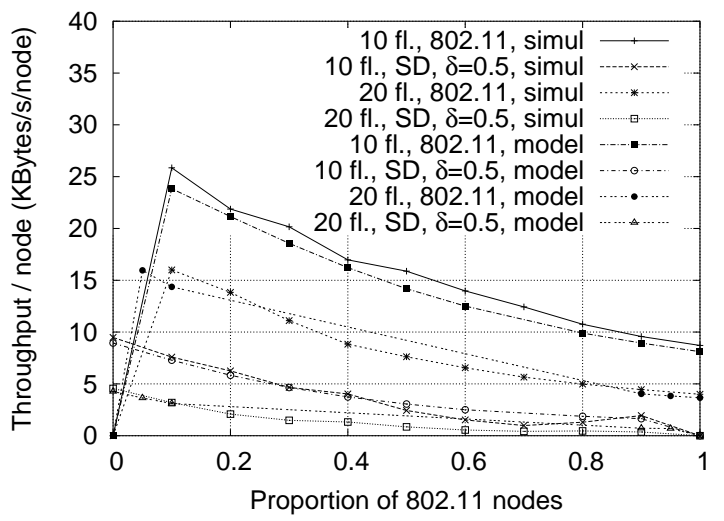

Fig. 14. Throughput of coexisting IEEE 802.11 and SD nodes

where:

$$
\begin{aligned}
& P_{t r 1}=\tau_{1} \\
& P_{s 1}=\left(1-\tau_{1}\right)^{\left(n_{1}-1\right)} \times\left(1-\tau_{2}\right)^{n_{2}} \\
& P_{t r 2}=\tau_{2} \\
& P_{s 2}=\left(1-\tau_{1}\right)^{n_{1}} \times\left(1-\tau_{2}\right)^{\left(n_{2}-1\right)} \\
& P_{t r}=1-\left(1-\tau_{1}\right)^{n_{1}} \times\left(1-\tau_{2}\right)^{n_{2}} \\
& P_{s}=\frac{n_{1} \tau_{1} P_{s 1}+n_{2} \tau_{2} P_{s 2}}{P_{t r}}
\end{aligned}
$$

In the following we keep the total number of flows using IEEE 802.11 and SD at a fixed value of 10 or 20 . Figure 14 shows the throughput of an IEEE 802.11 node and an SD node when the proportion of IEEE 802.11 nodes varies, based on the above model and the simulation results. Nodes that use SD have high CW values, trying to avoid collisions at high congestion levels. This is not the case of 802.11 that has relatively smaller CWs and keeps severe contention and less collision avoidance. Obviously, this results in an unequal share of the available channel bandwidth, i.e., 802.11 nodes are more aggressive than SD nodes. In these cases, 802.11 nodes "steal" some channel bandwidth from the SD nodes. Another important observation is that the average throughput is higher if all the stations use SD than if all of them use 802.11 protocol.
The above results show that it is better not to use SD as soon as one regular IEEE 802.11 station is present. The following mechanism can be used to decide whether to use SD. In the case of infrastructure mode, each SD station informs the AP that it is SD-compliant (using an extended Probe Response Frame), then the AP can decide, based on the current information received, whether the SD mode can be used in the next beacon interval. In other words, stations use SD only if explicitly mentioned by the $\mathrm{AP}$ in the beacon (denoted by extended beacon format). In the case of ad-hoc mode, beacon generation is distributed between each station. If one station does not send an extended beacon format, then all the SD stations will decide to switch back to IEEE 802.11 operation mode but they will continue to report their SD compliance in their beacon. If no more regular beacons are received after some timeout, SD stations can decide to use SD again. Actually the use of the beacon to send a specific option has already been proposed in the standard to force IEEE 802.11g back to IEEE 802.11b in a mixed IEEE 802.11b/g environment.

\section{$6 \quad$ Energy saving}

When the congestion level is high, frames are most likely to collide and be retransmitted before reaching their destinations successfully. The energy consumption at the sender, as well as at the receiver, is therefore proportional to the number of retransmissions. Slowly decreasing CWs, as in our scheme, reduces the risks of collisions and the corresponding retransmissions, for the same number of successful receptions, saving considerable energy. We simulate a scenario where we have $n$ flows each with 1 MBytes of data to transfer (using FTP/TCP), without RTS/CTS, considering that the transmission power is $600 \mathrm{~mW}$ and the receiving power is $300 \mathrm{~mW}$. The average 


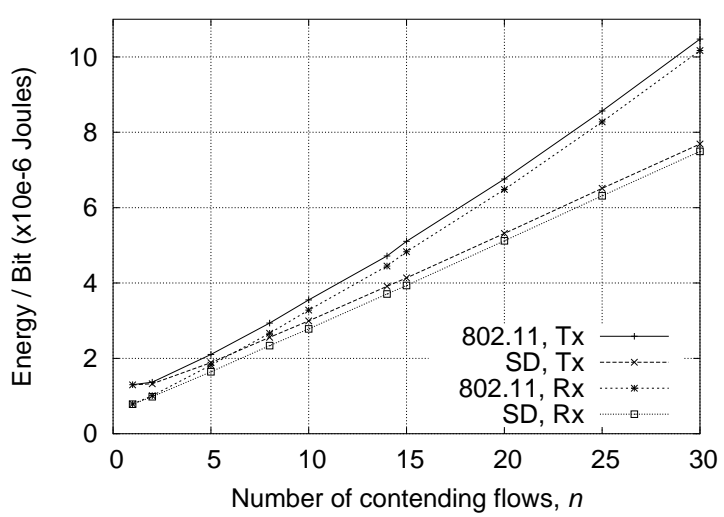

Fig. 15. Throughput energy consumption

energy per successfully received bit is shown in Figure 15.

The energy curves shown here are an image of the number of retransmissions for each successfully received frame. When the number of contending flows increases, the collision rate increases, spending more energy to deliver a good frame. However this is considerably lower with SD than with IEEE 802.11, due to the better adapted $\mathrm{CW}$ values. At low numbers of contending flows, no considerable collisions occur, therefore the energy consumption per successfully received packet is the same for IEEE 802.11 and for SD.

Reducing the number of retransmissions, by avoiding collisions, not only reduces the energy consumption but the total data transfer duration too. The overhead introduced by SD becomes negligible when collisions and retransmissions occur. Figure 16 shows that, when 30 flows contend to transmit 1 MBytes each, it takes 270 seconds for IEEE 802.11 to achieve the transfer. It takes considerably less (200 seconds) for SD to do the same job.

This duration difference between SD and IEEE 802.11 decreases with the number of contending flows. We should note that at higher numbers of contending flows, we start observing long TCP timeouts for some flows, causing disconnections, and reconnections at later times, whether for SD or IEEE 802.11. This makes the FTP duration measurement considered here inappropriate for a very high number of flows.

\section{$7 \quad$ Noisy channels}

Both IEEE 802.11 and SD suffer from a common problem in noisy channels: They cannot distinguish noise lost frames from collision lost frames. In both situations a node does not receive its frame ACK and doubles its CW to avoid further collisions, which is not needed if the frame was noise dropped. This adds an overhead that, in addition to the noise dropped frames, reduces the throughput considerably. This can be observed in Figure 17. For all values of packet error rate (PER) we can see that the throughput drop is much larger than the corresponding PER, because of the useless $\mathrm{CW}$ increase caused by noise. For instance at $P E R=0.1$, for a single flow, the throughput drop is (203007 to 123522 ) 39\% although only $10 \%$ of the packets are corrupted. ${ }^{5}$ The effect of noise lost frames is even more harmful

5 For the case of four flows in Figure 17, at $P E R>0.05$, we can see that the throughput is higher than that of a single flow. This is due to the fact that the aggregated throughput of the flows, reduced by $10 \%$, observes no considerable collisions that may reduce it.

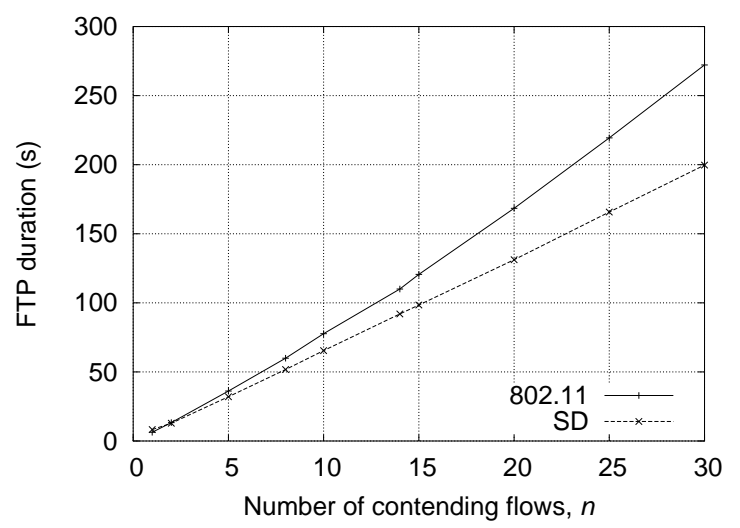

Fig. 16. FTP duration comparison 


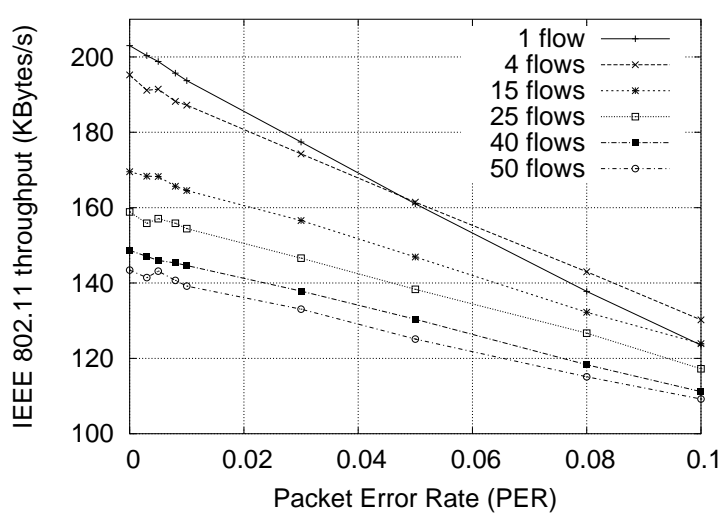

Fig. 17. Throughput of IEEE 802.11 in noisy channel

to $\mathrm{SD}$ since it causes CWs to get high, without necessarily avoiding any collisions. More precisely, both IEEE 802.11 and SD would increase the same way, but SD CWs decrease more slowly, adding more overhead, and wasting bandwidth, unless collision also exists.

Figure 18 shows the throughput gain of SD over IEEE 802.11 when varying the PER and the number of contending flows, without RTS/CTS. For a single flow that accesses the channel, the throughput of SD stays close to that of IEEE 802.11 as long as the PER is $<0.01$. Beyond this point (very severe channel conditions) the frequently corrupted frames keep the CWs relatively high with SD, and the gain decreases. The gain increases with the number of competing flows. At high PER, frequently corrupted frames still cause CWs to stay high, but to the advantage of avoiding collisions in this case.

\section{Implementation costs}

Among several implementation issues we discuss those specific to the SD mechanism: computation of contention windows and random number generation at the lowest chip level. Other implementation issues of the current

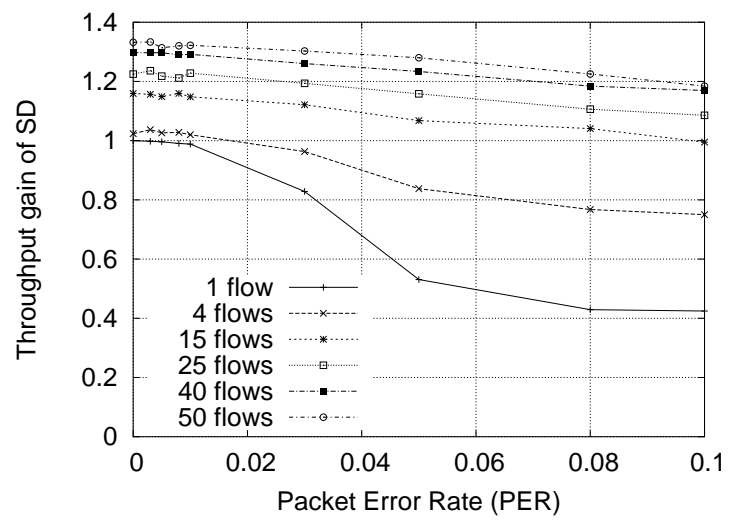

Fig. 18. Throughput gain of SD over IEEE 802.11 in noisy channel

standard are out of scope of this paper.

Upon a successful transmission, a new CW value is computed by multiplying the old value by $\delta<1$. By decomposing $\delta$ into powers of $1 / 2$, i.e. $\delta=\sum_{i=1}^{\infty} c_{i}(1 / 2)^{i}$, the SD mechanism consists of register shifts and additions of the previous CW value.

The number of register shifts (divisions) and additions depend on the chosen value of $\delta$. For instance $\delta=1 / 2+1 / 4=0.75$ needs two register shifts and one addition in order to compute the new CW value. Limiting the decomposition of $\delta$ to a small number of powers of $(1 / 2)$ reduces complexity. Futhermore, the use of lookup tables, with pre-computed CW values avoids real-time computations (at each successful transmission), making it as costly as the current CW reset mechanism.

The new CW value is used to generate the random backoff time. Implementation mandates the use of power of 2 random generators (in $\left.\left[0,2^{n}-1\right]\right)$. Therefore, since a CW with SD is not necessarily a power of 2 , an additional multiplication and a division (by $2^{m}$ ) are added to the current standard random number generation, as follows:

$$
b k f=(v \times C W) / 2^{m}
$$


where $v$ is a randomly generated number uniformly distributed in $\left[0,2^{m}\right]$ and $m=\left\lceil\log _{2}\left(C W_{\max }\right)\right\rceil$. Therefore, one multiplication and one register $m$-shift are added to the cost of the current standard.

\section{Acknowledgment}

The authors would like to thank Prof. Giuseppe Bianchi and Ms. Ilenia Tinnirello from Università di Palermo, Italy, for their valuable help.

\section{References}

\section{Conclusion}

In this paper we investigated slow $\mathrm{CW}$ decrease (SD) instead of CW reset after each successful frame transmission. This avoids future collisions, considering that the congestion level is unlikely to drop suddenly. It also reduces the number of frame retransmissions (which would also reduce the congestion on the channel), increasing the throughput considerably, decreasing delays and jitters. It performs as good as IEEE 802.11 in non-congested environments, and shows considerable gain over the latter in congested ones. The throughput gain is a function of frame lengths and data rates. We showed through simulations and mathematical modeling the considerable gain when using large data frames (37\%), and we extended the analysis for the worst gain values, that is for short data frames, e.g. when using RTS/CTS. Multiplicative CW decrease functions showed high throughput gains, with relatively low settling times after sudden congestion level drops. Fairness and coexistence between SD and 802.11 were explored, showing the weak points and their solutions as well. The effect of channel noise on SD and its considerable power saving were also analyzed. Last, we evaluated the implementation cost of the SD mechanism. Future work includes adaptive CW decrease algorithms in which decrease parameters change with the congestion load level in order to further enhance the SD performances.
[1] B. P. Crow, I. Widjaja, J. G. Kim, and P. T. Sakai, "IEEE 802.11 wireless local area networks," IEEE Communication magazine, September 1997.

[2] B. O'Hara and A. Petrick, IEEE 802.11 handbook. A designer's companion. IEEE Press, 1999.

[3] L. S. Committee, IEEE Std 802.11:Wireless LAN Medium Access Control (MAC) and Physical Layer (PHY) Specifications. IEEE Computer Society, 1999.

[4] L. Kleinrock and F. A. Tobagi, "Packet Switching in Radio Channels: Part 1: CSMA Modes and Their Throughput Delay Characteristics." IEEE Trans. on Comm. COM-23, 1975.

[5] V. Bharghavan, A. Demers, S. Shenker, and L. Zhang, "MACAW: A media acess protocol for wireless LANs," in Proceedings of ACM Sigcomm, 1994.

[6] P. Karn, "MACA: A new channel access method for packet radio." in $A R R L / C R R L$ Amateur radio 9th computer networking conference, 1990.

[7] T. Nandagopal, T.-E. Kim, X. Gao, and V. Bharghavan, "Achieving MAC layer fairness in wireless packet networks." in Proceedings of Mobicom, 2000.

[8] T. Ozugur, M. Naghshineh, K. Parviz, M. Olsen, B. Rezvani, and J. Copeland, "Balanced media access methods for wireless networks," in Proceedings of Mobicom, 1998. 
[9] The VINT Project, "Network Simulator", http://www.isi.edu/nsnam/ns/

[10] G. Bianchi, "Performance analysis of the IEEE 802.11 distributed coordination function," IEEE journal on selected areas in communications, vol. 18, 2000.

[11] G. Bianchi and I. Tinnirello, "Kalman Filter Estimation of the Number of Competing Terminals in an IEEE 802.11 network," in Proceedings of IEEE Infocom, 2003.

[12] R. K. Jain, D.-M. W. Chiu, and W. R. Hawe, "A quantitative measure of fairness and discrimination for resource allocation in shared computer system," Digital Equipment Corporation, Tech. Rep., 1984.

[13] C. E. Koksal, H. Kassab, and H. Balakrishnan, "An analysis of short-term fairness in wireless media access protocols," in Proceedings of ACM Sigmetrics, 2000.

[14] Q. Ni, I. Aad, C. Barakat, and T. Turletti, "Modelling and analysis of slow CW decrease for IEEE 802.11 WLAN," in Proceedings of IEEE International Symposium on Personnel, Indoor and Mobile Radio Communications (PIMRC 2003), Beijing, China, September 2003.

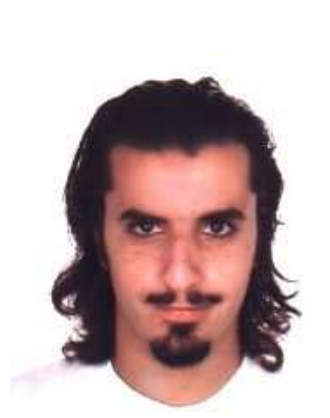

Imad Aad is a senior researcher at EPFL, Switzerland, working within the LCA team. $\mathrm{He}$ got his Electrical and Electronics engineering degree in 1998 from the Lebanese University, Beirut. He got his M.S. degree in 1999 from the University of Nice Sophia Antipolis, then his Ph.D. degree from Joseph Fourier University, France, in 2003. He prepared his Ph.D. on quality of service in wireless LANs at INRIA, France, within the Planète group. He joined EPFL in June 2003 where he is working now on cheating and cheating detection issues, DoS attacks and resilience and on general security aspects in wireless networks.

E-mail: imad.aad@epfl.ch

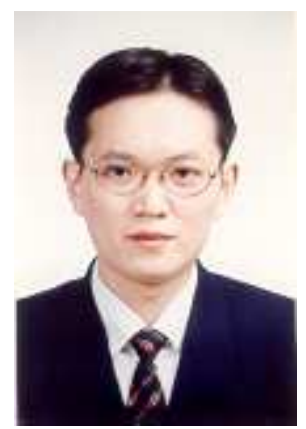

Qiang Ni received the B.S., M.S. and Ph.D. degrees from Hua Zhong University of Science and Technology (HUST), Wuhan City, China in 1993, 1996 and 1999 respectively. From 1999 to 2001 , he was a postdoctoral research fellow in the multimedia and wireless communication laboratory, HUST. He did his research internship at wireless and networking group of Microsoft Research Asia during the year of 2000. In 2001, he joined INRIA, where he was an expert research engineer at Planète group, France. He is currently a senior research scientist at Hamilton Institute, National University of Ireland Maynooth. Since 2002 he has been active as a voting member for the IEEE 802.11 wireless LAN Standard Working Group. He has served as Technical Program Committee member for the IEEE Vehicular Technology Conference, Integrated Heterogeneous Wireless Networks Symposium, 2003. His current research interests include communication protocol design and analysis for wireless LAN/PAN/MAN and 4G networks, vertical handover and mobility management in mobile networks, and multimedia transmission over hybrid wired/wireless networks.

E-mail: Qiang.Ni@ieee.org 


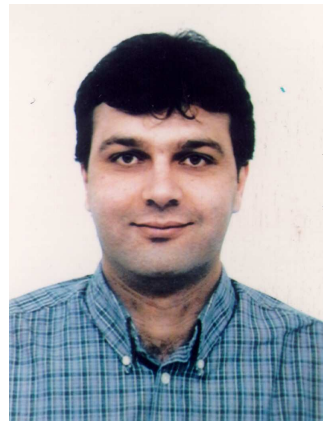

Chadi Barakat is a permanent research scientist in the Planète research group at INRIA - Sophia Antipolis since March 2002. He got his Electrical and Electronics engineering degree from the Lebanese University of Beirut in 1997, and his master and Ph.D. degrees in Networking from the University of Nice - Sophia Antipolis in 1998 and 2001. His Ph.D. has been done in the Mistral group at INRIA - Sophia Antipolis. From April 2001 to March 2002, he was with the LCA department at EPFL-Lausanne for a post-doctoral position, and from March to August 2004 he was a visiting faculty member at Intel Research Cambridge. Chadi Barakat was the general chair of PAM 2004 and serves in the program committees of many international conferences as Infocom, PAM, WONS, ASWN and Globecom. His main research interests are congestion and error control in computer networks, the TCP protocol, voice over IP, wireless LANs, Internet measurement and traffic analysis, and performance evaluation of communication protocols.

E-mail: Chadi.Barakat@sophia.inria.fr

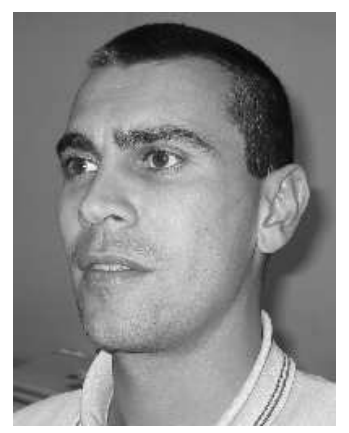

Thierry Turletti received the M.S. (1990) and the Ph.D. (1995) degrees in computer science both from the University of Nice - Sophia Antipolis, France. He has done his $\mathrm{PhD}$ studies in the RODEO group at INRIA Sophia Antipolis. During the year 199596, he was a postdoctoral fellow in the Telemedia, Networks and Systems group at LCS, MIT. He is currently a research scientist at the Planète group at INRIA Sophia Antipo- lis. His research interests include multimedia applications, congestion control and wireless networking. Dr. Turletti currently serves on the Editorial Board of Wireless Communications and Mobile Computing.

E-mail: Thierry.Turletti@sophia.inria.fr 